Check for updates

Cite this: Chem. Commun., 2017, 53, 9793

Received 11th June 2017,

Accepted 9th August 2017

DOI: $10.1039 / c 7 c c 04525 e$

rsc.li/chemcomm

\section{Supramolecular core-glycoshell polythiophene nanodots for targeted imaging and photodynamic therapy $\dagger$}

\author{
Hai-Hao Han, ${ }^{\mathrm{ab}}$ Chang-Zheng Wang, ${ }^{\mathrm{a}}$ Yi Zang, ${ }^{\mathrm{b}}$ Jia Li, ${ }^{\mathrm{b}}{ }^{\mathrm{b}}$ Tony D. James (D) ${ }^{\mathrm{c}}$ and \\ Xiao-Peng $\mathrm{He}^{\star a}$
}

\begin{abstract}
Supramolecular self-assembly between poly(3-hexylthiophene-2,5-diyl) (P3HT), a polymeric material extensively used for optoelectronic devices, and fluorescent glycoprobes produces core-glycoshell theranostic nanodots (glyco-dots) capable of targeted imaging and photodynamic therapy of liver and triple-negative breast cancer cells.
\end{abstract}

Theranostic systems that incorporate the ability to simultaneously detect and treat a disease offer exceptional opportunities for improved disease therapy. To minimise side-effects and enhance therapeutic efficiency, the introduction of a targeting group that can actively localise theranostic materials within pathologic tissues has been a popular strategy. ${ }^{1-5}$ The targeting groups developed include antibodies, peptides, aptamers and small molecules. ${ }^{6,7}$ With an increasing awareness that intercellular carbohydratereceptor interactions (CRIs) are crucial for the initiation of a number of human diseases, ${ }^{8-10}$ a variety of glycoligands have been employed as the targeting agent for disease imaging and therapy. ${ }^{11-19}$

In general, carbohydrates require a multivalent presentation on a backbone material to enhance the binding avidity for receptors because of the low affinity of monovalent CRIs. To overcome this problem, a number of elegant glycoclusters, glycodendrimers and other glycopolymeric materials have been developed for high-affinity binding with carbohydrate receptors. ${ }^{20}$ However, the production of these multivalent glycoconjugates often requires multiple synthetic and purification steps, thereby increasing the complexity for further functionalisation with theranostic partners. Here we report on the simple construction of core-glycoshell theranostic dots by the supramolecular

\footnotetext{
${ }^{a}$ Key Laboratory for Advanced Materials \& Institute of Fine Chemicals, East China University of Science and Technology, 130 Meilong Rd., Shanghai 200237, P. R. China.E-mail: xphe@ecust.edu.cn

${ }^{b}$ National Center for Drug Screening, State Key Laboratory of Drug Research, Shanghai Institute of Materia Medica, Chinese Academy of Sciences, 189 Guo Shoujing Rd., Shanghai 201203, P. R. China. E-mail: jli@simm.ac.cn ${ }^{c}$ Department of Chemistry, University of Bath, Bath, BA2 7AY, UK

$\dagger$ Electronic supplementary information (ESI) available: Additional figures and experimental section. See DOI: 10.1039/c7cc04525e
}

self-assembly between fluorescent glycoprobes (as both an imaging and a targeting agent) and poly(3-hexylthiophene-2,5diyl) (P3HT) nanodots (as both a vector and a therapeutic material for photodynamic therapy) (Fig. 1a). Although P3HT is among the most popular polymers in the construction of photovoltaic devices, ${ }^{21}$ its potential for biomedical applications has to date hardly been explored. ${ }^{22}$

To the best of our knowledge, with this research we have developed the first P3HT based core-glycoshell theranostic nanomaterial for cancer cells. We found that P3HT nanodots could self-assemble with the hydrophobic-dye moiety of the glycoprobes under aqueous conditions, producing multivalent glyco-dots (Fig. 1b). These simple glyco-dots have been shown to be amenable for targeted imaging of cancer cells that overly express carbohydrate receptors. Subsequent exposure of the cells to light significantly supresses the cell viability due to the release of reactive oxygen species (ROS) from the nanodots (Fig. 1c).

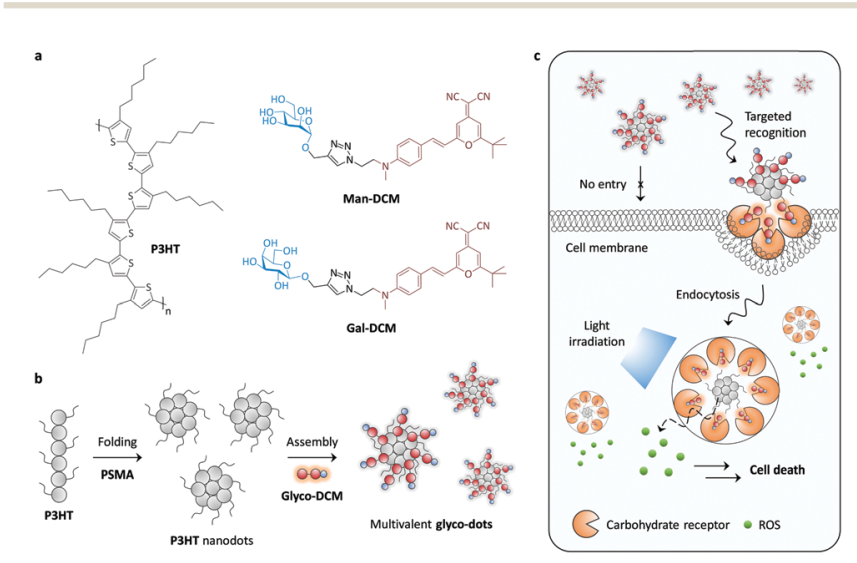

Fig. 1 (a) Structure of P3HT and the glycoprobes Man-DCM and Gal-DCM. (b) Schematic illustration of folding of P3HT to produce P3HT nanodots and the subsequent supramolecular self-assembly with the glycoprobes. (c) Schematic illustration of the targeted theranostic effect of glyco-dots based on selective carbohydrate-receptor interactions and the intracellular production of ROS upon light irradiation. 
Galactose (Gal) and mannose (Man), which can be selectively recognised by the asialoglycoprotein receptor (ASGPr; highly expressed on hepatic cells) ${ }^{23}$ and mannose receptor (MR; highly expressed on a breast cancer cell line), ${ }^{24}$ respectively, were coupled with a red-emitting dicyanomethylene- $4 H$-pyran (DCM) dye using the click reaction, to produce Gal-DCM and Man-DCM probes. ${ }^{25}$ In order to obtain stable and water-dispersible P3HT nanodots, P3HT was mixed with poly(styrene-co-maleic anhydride) (PSMA) $(20 \% \mathrm{w} / \mathrm{w})$, which were then folded to form the dots through a reprecipitation method. ${ }^{26}$ The core-shell glyco-dots were subsequently produced by the self-assembly of Gal-DCM and Man-DCM with the P3HT nanodots in Tris-HCl (0.01 M, pH 7.4), producing Gal-dot and Man-dot, respectively. The driving force by which the glyco-dots are formed is probably the result of $\pi$-stacking between the DCM moiety of the glycoprobes and the exposed hydrophobic surfaces of the polymeric backbone of the nanodots. ${ }^{27-29}$

A variety of techniques were used to characterise the glycodots. Transmission electron microscopy (TEM) images indicate that the assembly of the glycoprobes with P3HT produced mono-dispersed nanoparticles without altering the morphology of the P3HT nanodots (Fig. 2a). The P3HT core appears to be a solid as observed from the TEM images shown in Fig. 2a. Since the P3HT core is produced by co-folding the P3HT polymer with $20 \%$ of PSMA (to enhance water solubility), hydrophobic cavities might exist, allowing the nanodots to accommodate hydrophobic molecules (such as dyes and drugs). ${ }^{27-29}$ Using UV-vis spectroscopy, we determined that the absorbance peak of both Gal-dot (Fig. 2b) and Man-dot (Fig. 2c) blue-shifted by ca. $20 \mathrm{~nm}$ with respect to the P3HT dots alone, suggesting a change of the conjugated structure for the DCM upon binding to the polymer backbone. $^{30}$ The particle size of Gal-dot (Fig. 2d) and Man-dot (Fig. 2e) was found via dynamic light scattering to be slightly larger than that of the P3HT dot alone, suggesting the formation of a glycoprobe-shell on a P3HT core. Meanwhile, the $\zeta$ potential of the glyco-dots (Fig. 2f and $\mathrm{g}$ for Gal-dot and Man-dot, respectively) decreased significantly with respect to that of the P3HT dot. These data suggest the successful supramolecular assembly between the glycoprobes and the P3HT dots.

We then evaluated the targeted cellular imaging ability of the glyco-dots for Hep-G2 (human liver cancer cell line that overly expresses ASGPr) and MDA-MB-231 (human triple-negative breast cancer cell line that overly expresses MR). A human cervical cancer cell line (HeLa) with minimal expression of both receptors was used as the control. High-content fluorescence microscopy was used for cell imaging and the fluorescence intensity was quantified using a Columbus analysis system. We determined that the incubation of Gal-dot with the cells only led to fluorescence in Hep-G2 and not in HeLa or MDA-MB-231 cells in a concentrationdependent manner (Fig. 3a). The fluorescence intensity produced in Hep-G2 was much higher than that in the other cells (Fig. 3b). Likewise, the treatment of the cells with Man-dot led to selective fluorescence in MDA-MB-231 cells (Fig. 3d) with a much lower fluorescence in HeLa and Hep-G2 cells (Fig. 3e). These results are in good agreement with the ASGPr and MR mRNA level of the cells (Fig. $3 \mathrm{c}$ and $\mathrm{f}$ for ASGPr and MR, respectively), as determined via a real-time quantitative polymerase chain reaction.
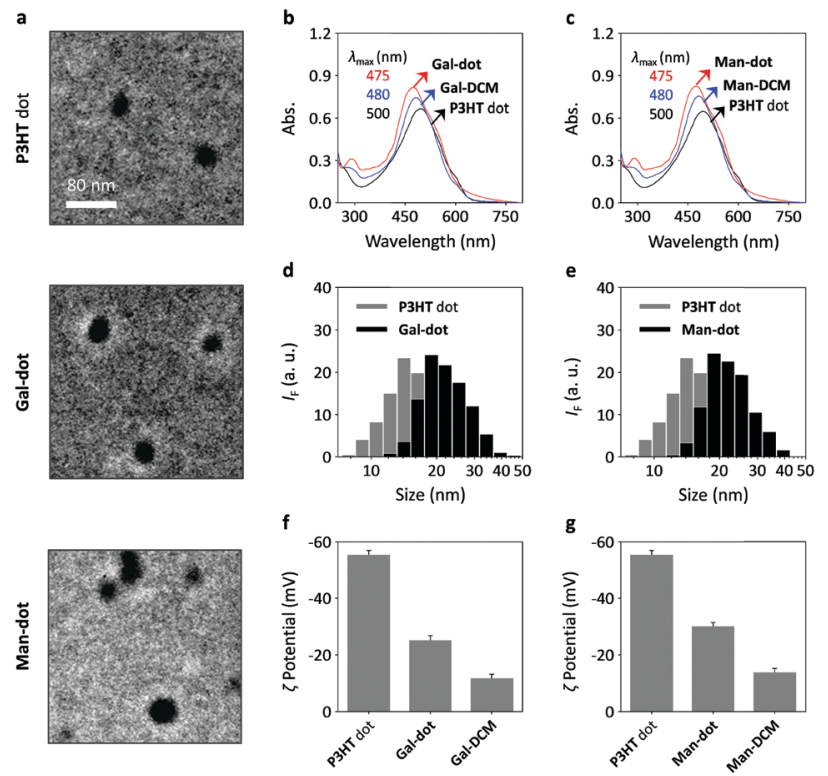

Fig. 2 (a) Transmission electron microscopy images of P3HT nanodot (100 ppm), Gal-dot (16 $\mu \mathrm{M} / 100 \mathrm{ppm})$ and Man-dot (16 $\mu \mathrm{M} / 100 \mathrm{ppm})$. Stacked UV-vis absorbance spectra of (b) Gal-DCM $(50 \mu \mathrm{M})$, P3HT nanodot (20 ppm) and Gal-dot (Gal-DCM/P3HT nanodots $=50 \mu \mathrm{M} / 20 \mathrm{ppm}$ ) and (c) Man-DCM (50 $\mu \mathrm{M})$, P3HT nanodots (20 ppm) and Man-dot (Man-DCM/P3HT nanodot $=50 \mu \mathrm{M} / 20 \mathrm{ppm}$ ) measured in Tris- $\mathrm{HCl}$ buffer (0.01 M, pH 7.4). Dynamic light scattering analysis of (d) P3HT nanodots (16 ppm) and Gal-dot (Gal-DCM/P3HT nanodots $=1 \mu \mathrm{M} / 16$ ppm) and (e) P3HT nanodots (16 ppm) and Man-dot (Man-DCM/P3HT nanodots = $1 \mu \mathrm{M} / 16 \mathrm{ppm}$ ) measured in Tris-HCl buffer (0.01 M, pH 7.4). Zeta potential of (f) Gal-DCM $(1 \mu \mathrm{M})$, P3HT nanodots (16 ppm) and Gal-dot (Gal-DCM/P3HT nanodots $=1 \mu \mathrm{M} / 16 \mathrm{ppm}$ ) and (g) Man-DCM (1 $\mu$ M), P3HT nanodots (16 ppm) and Man-dot (Man-DCM/P3HT nanodots $=1 \mu \mathrm{M} / 16 \mathrm{ppm}$ ) measured in deionized water.

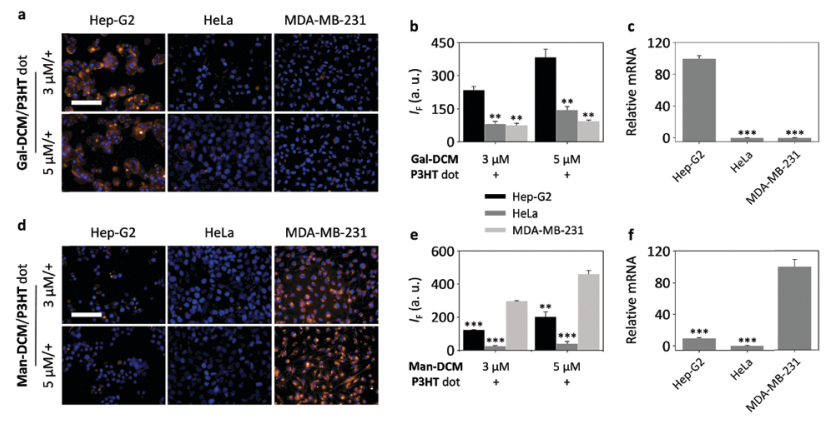

Fig. 3 Fluorescence imaging (a) and quantification (b) of Hep-G2 (human liver cancer), HeLa (human cervical cancer) and MDA-MB-231 (human triple-negative breast cancer) cells with Gal-dot (Gal-DCM $=3$ or $5 \mu \mathrm{M}$; P3HT nanodots $=16 \mathrm{ppm}$ ) of different concentrations. Fluorescence imaging (d) and quantification (e) of Hep-G2, HeLa and MDA-MB-231 cells with Man-dot (Man-DCM $=3$ or $5 \mu \mathrm{M}$; P3HT nanodots $=16 \mathrm{ppm}$ ) of different concentrations. Relative mRNA level of (c) ASGPr and (f) MR in Hep-G2, HeLa and MB-231. For fluorescence imaging, the excitation and emission wavelengths for DCM are $460-490 \mathrm{~nm}$ and $560-630 \mathrm{~nm}$, respectively (scale bar $=100 \mu \mathrm{m} ;{ }^{* \star} P<0.01 ;{ }^{* \star *} P<0.005$ ). Cell nuclei were stained by Hoechst 33342.

In addition, we determined that P3HT dots alone were not fluorescent by excitation at $480 \mathrm{~nm}$ (Fig. S1a, ESI $\dagger$ ), and the fluorescence of Gal-dot and Man-dot was significantly lower 
than that of Gal-DCM (Fig. S1b, ESI $\dagger$ ) and Man-DCM (Fig. S1c, ESI $\dagger$ ), respectively. The decreased fluorescence is suggestive of the quenching effect of conjugated polymers with closely attached fluorescent dyes, ${ }^{28,29}$ demonstrating the efficient assembly of the glycoprobes with the P3HT nanodots.

A titration assay was then carried out in order to examine the multivalent effect of the glyco-dots. The cells were treated with glycoprobes alone (monovalent) and then with the glyco-dots (multivalent), and the resulting images were compared qualitatively and quantitatively. We determined that the fluorescence intensity of Gal-DCM and Man-DCM increased gradually with increasing P3HT nanodots (Fig. S2a and b, ESI $\dagger$ ). The fluorescence enhancement reached equilibrium with a P3HT concentration of $24 \mathrm{ppm}$. This suggests that the multivalent nature of the glycoprobes on the P3HT nanodots can increase the avidity for the cell-surface receptors. This is in accordance with our previous observations using two-dimensional materials as a substrate to produce multivalency. ${ }^{16,25,31}$ To corroborate that the selective imaging was a result of CRIs, a competition assay was carried out. We found that the pre-incubation of free D-galactose and D-mannose with Hep-G2 and MDA-MB-231 (Fig. S2c and d, ESI $\dagger$ ) led to a concentration-dependent fluorescence suppression of Gal-dot and Man-dot, respectively. This suggests that the selective imaging effect of the glyco-dots was predominantly caused by carbohydrate-receptor recognition.

Having evaluated the nanodot targeted imaging ability, we further tested the photodynamic therapeutic effect of the glyco-dots by the treatment of the cells with light. Using Ce6 (a commercial photosensitizer) as a reference compound, we first determined that the treatment of the P3HT nanodot as well as the glyco-dot solution with light irradiation produced ROS in a time-dependent manner, as measured using a known fluorogenic ROS probe (Fig. S3a, ESI $\dagger$ ). The ROS production of the materials was also corroborated by DPA (a widely used singlet oxygen trapper) (Fig. S3b, ESI $\dagger$ ). We then employed a doublestaining assay to measure the cell death upon the treatment of the cells in the absence and presence of glyco-dots with light. Hoechst 33342 and Sytox Green were used to stain the total and dead cells, respectively. ${ }^{32}$ We observed that Gal-dot with light irradiation caused increased cell death of Hep-G2 (Fig. 4a and b). In contrast, the presence of Gal-dot without light irradiation or irradiation of Hep-G2 without Gal-dot did not lead to cell death. Likewise, although the treatment of MDA-MB-231 with just light or Man-dot caused minimal cell death, the presence of both led to an increase in dead MDA-MB-231 cells (Fig. 4a and $b$ ). We also determined that the light irradiation of HeLa cells pre-treated with both glyco-dots resulted in minimal cell death. A subsequent cell viability assay indicated that, upon light irradiation, the viabilities of Hep-G2 and MDA-MB-231 were supressed in the presence of Gal-dot and Man-dot, respectively, whereas those of HeLa cells were hardly affected (Fig. 4c).

The cell viabilities of Hep-G2, MDA-MB-231, HeLa as well as a healthy cell line 293T (human embryonic kidney) were tested with increasing P3HT nanodots and the glyco-dots in the presence or absence of light irradiation. The results (Fig. S4, ESI $\dagger$ ) suggest that (1) the P3HT nanodot alone has low cytotoxicity for all the
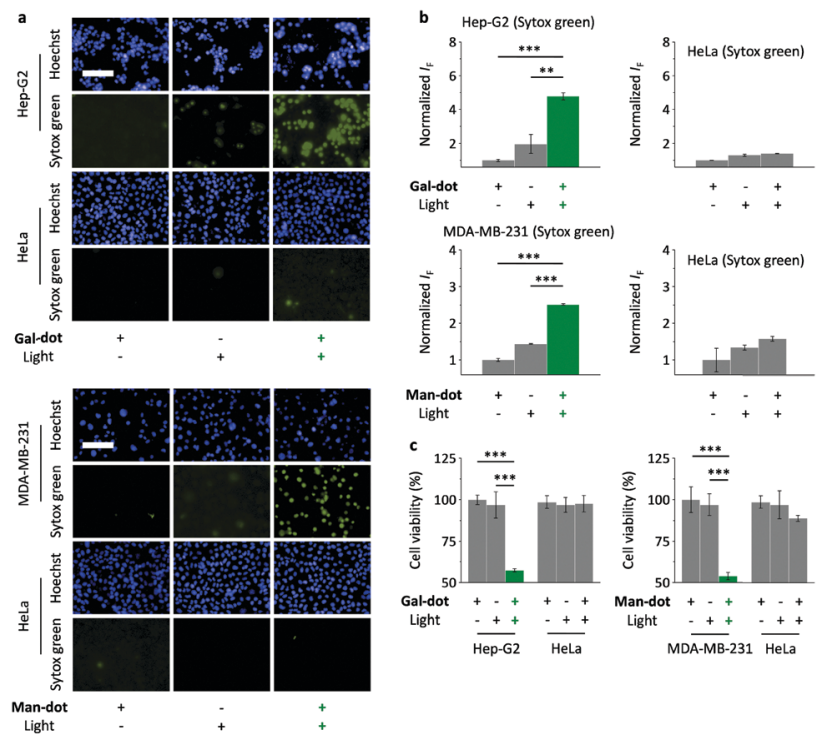

Fig. 4 Fluorescence imaging (a) and quantification (b) of Hep-G2, HeLa and MDA-MB-231 cells treated with Gal-dot (Gal-DCM/P3HT nanodots = $5 \mu \mathrm{M} / 16 \mathrm{ppm}$ ) and Man-dot (Man-DCM/P3HT nanodots $=5 \mu \mathrm{M} / 16 \mathrm{ppm}$ ) with or without light irradiation, as double-stained by Hoechst 33342 and Sytox Green. (c) Viabilities of Hep-G2, HeLa and MDA-MB-231 cells treated with Gal-dot (Gal-DCM/P3HT nanodots $=5 \mu \mathrm{M} / 16 \mathrm{ppm}$ ) and Man-dot (Man-DCM/P3HT nanodots $=5 \mu \mathrm{M} / 16 \mathrm{ppm}$ ) with or without light irradiation. For fluorescence imaging, the excitation and emission wavelengths for Sytox Green are $490-510 \mathrm{~nm}$ and $530-590 \mathrm{~nm}$, respectively (scale bar $=100 \mu \mathrm{m}$; $\left.{ }^{\star \star} P<0.01 ;{ }^{* \star *} P<0.005\right)$

cells tested irrespective of light irradiation, demonstrating the importance of the glyco-shell to selectively target a transmembrane receptor, and enhance the targeted photodynamic effect, (2) Gal-dot and Man-dot resulted in selective cell death of Hep-G2 and MDA-MB-231 in a concentration-dependent manner, and (3) the nanomaterials are not toxic to a healthy cell line (293T) irrespective of light irradiation. These data clearly demonstrate the targeted theranostic potential of the glyco-dots. Also, we have performed standard cell viability assays demonstrating that the nanomaterials hardly affect the proliferation of both cancer and healthy cells used in this study (Fig. S5, ESI $\dagger$ ).

We have developed a simple strategy based on the supramolecular self-assembly between glycoprobes and P3HT that can form nano-sized, mono-dispersed polymer nanodots in a fully aqueous solution. The self-assembled core-shell glycodots showed high avidity for transmembrane carbohydrate receptors probably due to a supramolecular multivalent presentation of the glycoprobes on the polymer backbone. These multivalent "glycoshell" systems can be used to target a specific cancer cell, resulting in fluorescence for cell imaging, and the polymer core kills the targeted cells via light-controlled release of a toxic level of ROS. This research paves the way towards the construction of materials capable of targeted disease theranostics using the versatile supramolecular functionalization of known polymeric materials.

This research was supported by the 973 project (2013CB733700), the National Natural Science Foundation of China (21572058), the Science and Technology Commission of Shanghai Municipality 
(15540723800), the Fundamental Research Funds for the Central Universities (222201717003) and the Shanghai RisingStar Program (16QA1401400) (to X.-P. He). Prof. Changfeng Wu is warmly thanked for helpful discussions. The Catalysis And Sensing for our Environment (CASE) network is thanked for research exchange opportunities. T. D. J. thanks ECUST for a guest professorship.

\section{Conflicts of interest}

There are no conflicts to declare.

\section{Notes and references}

1 K. Yang, L. Feng, X. Shi and Z. Liu, Chem. Soc. Rev., 2013, 42, 530-537. 2 T. Lammers, S. Aime, W. E. Hennink, G. Storm and F. Kiessling, Acc. Chem. Res., 2011, 44, 1029-1038.

3 W. T. Al-Jamal and K. Kostarelos, Acc. Chem. Res., 2011, 44, 1094-1104. 4 K. Y. Choi, G. Liu, S. Lee and X. Chen, Nanoscale, 2012, 4, 330-342. 5 Y.-P. Ho and K. W. Leong, Nanoscale, 2010, 2, 60-68.

6 M. K. Yu, J. Park and S. Jon, Theranostics, 2012, 2, 3-44.

7 S. S. Kelkar and T. M. Reineke, Bioconjugate Chem., 2011, 22, 1879-1903.

8 C. R. Bertozzi and L. L. Kiessling, Science, 2001, 291, 2357-2364.

9 T. Feizi and W. Chai, Nat. Rev. Mol. Cell Biol., 2004, 5, 582-588.

10 F.-T. Liu and G. A. Rabinovich, Nat. Rev. Cancer, 2005, 5, 29-41.

11 For a recent feature article, see: X.-P. He, Y. Zang, T. D. James, J. Li, G.-R. Chen and J. Xie, Chem. Commun., 2017, 53, 82-90.

12 For a recent mini-review article, see: X.-P. He, Y.-L. Zeng, Y. Zang, J. Li, R. A. Field and G.-R. Chen, Carbohydr. Res., 2016, 429, 1-22.

13 M. Lin, Y. Zhang, G. Chen and M. Jiang, Small, 2015, 11, 6065-6070.

14 B. R. Schroeder, M. I. Ghare, C. Bhattacharya, R. Paul, Z. Yu, P. A. Zaleski, T. C. Bozeman, M. J. Rishel and S. M. Hecht, J. Am. Chem. Soc., 2014, 136, 13641-13656.

15 (a) X. Wu, M. Yu, B. Lin, H. Xing, J. Han and S. Han, Chem. Sci., 2015, 6, 798-803; (b) X. Wu, M. Yu, B. Lin, H. Xing, J. Han and S. Han, Chem. Sci., 2015, 6, 2002-2009.

16 (a) H.-L. Zhang, X.-L. Wei, Y. Zang, J.-Y. Cao, S. Liu, X.-P. He, Q. Chen, Y.-T. Long, J. Li, G.-R. Chen and K. Chen, Adv. Mater., 2013, 25, 4097-4101; (b) D.-L. Ji, Y. Zhang, Y. Zang, J. Li, G.-R. Chen, X.-P. He and H. Tian, Adv. Mater., 2016, 28, 9356-9363.

17 (a) X.-P. He, Y.-L. Zeng, X.-Y. Tang, N. Li, D.-M. Zhou, G.-R. Chen and H. Tian, Angew. Chem., Int. Ed., 2016, 55, 13995-13999; (b) K.-B. Li, Y. Zang, H. Wang, J. Li, G.-R. Chen, T. D. James, X.-P. He and
H. Tian, Chem. Commun., 2014, 50, 11735-11737; (c) L. Dong, Y. Zang, D. Zhou, X.-P. He, G.-R. Chen, T. D. James and J. Li, Chem. Commun., 2015, 51, 11852-11855; (d) D.-T. Shi, D. Zhou, Y. Zang, J. Li, G.-R. Chen, T. D. James, X.-P. He and H. Tian, Chem. Commun., 2015, 51, 3653-3655; (e) D. Xie, D.-K. Ji, Y. Zhang, J. Cao, H. Zheng, L. Liu, Y. Zang, J. Li, G.-R. Chen, T. D. James and X.-P. He, Chem. Commun., 2016, 52, 9418-9421; $(f)$ M. Wahiba, X.-Q. Feng, Y. Zang, T. D. James, J. Li, G.-R. Chen and X.-P. He, Chem. Commun., 2016, 52, 11689-11692; $(g)$ N. Chen, Z.-H. Yu, D. Zhou, X.-L. Hu, Y. Zang, X.-P. He, J. Li and J. Xie, Chem. Commun., 2016, 52, 2284-2287; (h) M. Zhang, P. Wu, W.-T. Dou, H.-H. Han, X.-P. He, C. Tan and Y. Jiang, Chem. Commun., 2017, 53, 5625-5628.

18 (a) X.-L. Hu, Y. Zang, J. Li, G.-R. Chen, T. D. James, X.-P. He and H. Tian, Chem. Sci., 2016, 7, 4004-4008; (b) K.-B. Li, N. Li, Y. Zang, G.-R. Chen, J. Li, T. D. James, X.-P. He and H. Tian, Chem. Sci., 2016, 7, 6325-6329.

19 J.-X. Song, X.-Y. Tang, D.-M. Zhou, W. Zhang, T. D. James, X.-P. He and H. Tian, Mater. Horiz., 2017, 4, 431-436.

20 For a recent comprehensive review, see: S. Cecioni, A. Imberty and S. Vidal, Chem. Rev., 2015, 115, 525-561.

21 M. T. Dang, L. Hirsch and G. Wantz, Adv. Mater., 2011, 23, 3597-3602.

22 (a) S. Vaquero, C. Bossio, S. Bellani, N. Martino, E. Zucchetti, G. Lanzani and M. R. Antognazza, J. Mater. Chem. B, 2016, 5, 5272-5283; (b) E. Zucchetti, M. Zangoli, I. Bargigia, C. Bossio, F. Di Maria, G. Barbarella, C. D'Andrea, G. Lanzani and M. R. Antognazza, J. Mater. Chem. B, 2017, 5, 565-574.

23 J. B. Burgess, J. U. Baenziger and W. R. Brown, Hepatology, 1992, 15, 702-706.

24 D. Brevet, M. Gary-Bobo, L. Raehm, S. Richeter, O. Hocine, K. Amro, B. Loock, P. Couleaud, C. Frochot, A. Morère, P. Maillard, M. Garcia and J.-O. Durand, Chem. Commun., 2009, 1475-1477.

25 D.-K. Ji, G.-R. Chen, X.-P. He and H. Tian, Adv. Funct. Mater., 2015, 25, 3483-3487.

26 C. Wu, C. Szymanski and J. McNeill, Langmuir, 2006, 22, 2956-2960.

27 C.-Z. Wang, J.-L. Chen, Y. Tang, Y. Zang, G.-R. Chen, T. D. James, J. Li, C. Wu and X.-P. He, ACS Appl. Mater. Interfaces, 2017, 9, 3272-3276.

28 W.-T. Dou, Y.-L. Zeng, Y. Lv, J. Wu, X.-P. He, G.-R. Chen and C. Tan, ACS Appl. Mater. Interfaces, 2016, 8, 13601-13606.

29 W.-T. Dou, Y. Zhang, Y. Lv, J. Wu, Y. Zang, C. Tan, J. Li, G.-R. Chen and X.-P. He, Chem. Commun., 2016, 52, 3821-3824.

30 N. Dogra, X. Li and P. Kohli, Langmuir, 2012, 28, 12989-12998.

31 (a) D.-K. Ji, Y. Zhang, X.-P. He and G.-R. Chen, J. Mater. Chem. B, 2015, 3, 6656-6661; (b) D.-K. Ji, Y. Zhang, Y. Zang, W. Liu, X. Zhang, J. Li, G.-R. Chen, T. D. James and X.-P. He, J. Mater. Chem. B, 2015, 3, 9182-9185.

32 J. Feng, T. Wang, S. Zhang, W. Shi and Y. Zhang, PLoS One, 2014, 9, e111809. 